Check for updates

The BMJ

Cite this as: $B M / 2020 ; 371: \mathrm{m} 3937$ http://dx.doi.org/10.1136/bmj.m3937 Published: 09 October 2020

\section{Covid-19: Experts advise cautious optimism for neutralising antibodies after early results}

\section{Elisabeth Mahase}

Pharmaceutical company Eli Lilly has submitted a request to the US Food and Drug Administration for emergency use authorisation of its bamlanivimab montherapy in higher risk patients who have recently been diagnosed with mild to moderate covid-19.

Bamlanivimab is a neutralising IgG1 monoclonal antibody (mAb) developed specifically to target the spike protein of SARS-CoV-2. It is designed to stop the virus entering human cells, so neutralising it, and potentially preventing and treating covid-19.

On 16 September Lilly announced that the treatment reduced the rate of hospitalisation and emergency department visits in nursing home residents and staff with mild to moderate covid-19 ( 5 in 302), when compared with placebo (9 in 150). ${ }^{1}$ The company has said that it could supply up to one million doses (700 $\mathrm{mg}$ ) of bamlanivimab between October and December 2020, with 100000 initially available in October.

Eli Lilly has also announced that the interim analysis of its Blaze-1 phase II clinical trial showed that a combination of two neutralising antibodies (bamlanivimab and etesevimab) reduced viral load, symptoms, and covid-19 related hospitalisation and emergency department visits in recently diagnosed patients with mild to moderate covid-19. ${ }^{2}$

The results for both the monotherapy and combination therapy have only been shared through a press release, however, without a preprint of a paper or a peer reviewed publication. The company said it will publish the results in "peer reviewed journals as soon as possible."

Experts have said that the reports seem promising but stressed that the studies were small.

"There is a way to go before we know whether this class of drugs is effective in reducing the worst manifestations of covid-19," said Martin Landray, professor of medicine and epidemiology at the University of Oxford and co-chief investigator of the Recovery trial.

The randomised, double blind, placebo controlled study for the combination therapy evaluated bamlanivimab and etesevimab, which bind to regions of the SARS-CoV-2 spike protein. Patients were assigned to receive either $2800 \mathrm{mg}$ of each antibody $(\mathrm{n}=112)$ or placebo $(\mathrm{n}=156)$.

According to Eli Lilly, the combination therapy significantly reduced viral load at day $11(\mathrm{P}=0.011)$, meeting the primary endpoint of the study-although most patients, including those receiving placebo, demonstrated near complete viral clearance by day 11.
However, an exploratory analysis showed that the proportion of patients with persistent high viral load at day seven for combination therapy was lower (3.0\%) $v$ placebo (20.8\%), corresponding to a nominal $\mathrm{P}$ value of $\mathrm{P}<0.0001$ without multiplicity adjustment.

Improvements in symptoms were observed as early as three days after dosing, while the rate of covid-19 related hospitalisation and emergency department visits was also lower for patients treated with combination therapy (0.9\%) $v$ placebo $(5.8 \%)$, a relative risk reduction of $84.5 \%(\mathrm{P}=0.049)$.

In terms of adverse events, the company reported that the therapy was "generally well tolerated with no drug related serious adverse events."

Stephen Evans, professor of pharmacoepidemiology at the London School of Hygiene and Tropical Medicine, said, "These are among the first drugs that have been specifically developed from scratch to work against the SARS-Cov-2 virus. The evidence for their efficacy is encouraging but is still limited"

Evans added that the process of applying for an emergency use authorisation (EUA) in the US "does not mean that the company has got sufficient data now for the EUA, but will hope to have such data very soon. Cautious optimism is justified.”

Eli Lilly said it plans to submit a subsequent request for EUA for combination therapy in November, "pending clinical trial enrolment, once additional safety data accumulate and sufficient supply is manufactured.”

A Study of LY3819253 (LY-CoV555) in preventing SARS-CoV-2 infection and covid-19 in nursing home residents and staff (Blaze-2). 2020.

2 A Study of LY3819253 (LY-CoV555) and LY3832479 (LY-CoV016) in participants with mild to moderate covid-19 illness (Blaze-1). 2020. https://clinicaltrials.gov/ct2/show/NCT04427501 https://clinicaltrials.gov/ct2/show/NCT04497987 